\title{
FOLIATIONS BY CURVES UNIQUELY DETERMINED BY MINIMAL SUBSCHEMES OF ITS SINGULARITIES
}

\author{
ANTONIO CAMPILLO AND JORGE OLIVARES
}

Dedicated to the memory of Professor Egbert Brieskorn

\begin{abstract}
It is well-known that a foliation by curves of degree greater than or equal to two, with isolated singularities, in the complex projective space of dimension greater than or equal to two, is uniquely determined by the scheme of its singular points. The main result in this paper is that the set of foliations which are uniquely determined by a subscheme (of the minimal possible degree) of its singular points, contains a nonempty Zariski-open subset. Our results hold in the projective space defined over any algebraically closed ground field.
\end{abstract}

\section{INTRODUCTION AND STATEMENT OF THE RESULTS}

Let $\mathbb{P}^{n}=\mathbb{P}_{\mathbf{K}}^{n}$ be the projective space of dimension $n \geq 2$ over an algebraically closed ground field $\mathbf{K}$ and let $\mathcal{O}_{\mathbb{P}^{n}}, \Theta_{\mathbb{P} n}$ and $\mathcal{H}$ denote its structure, tangent and hyperplane sheaves. For an

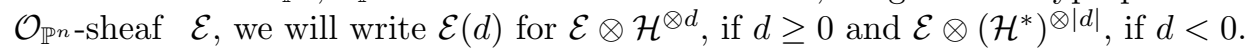

Let

$$
\mathbf{E}=\mathbf{E}(n, r-1)=\mathrm{H}^{0}\left(\mathbb{P}^{n}, \Theta_{\mathbb{P}^{n}}(r-1)\right), \text { and } \mathbf{e}=\mathbf{e}(n, r-1)=\operatorname{dim}_{\mathbf{K}} \mathbf{E} .
$$

A foliation by curves with singularities (or simply a foliation in the sequel) of degree $r$ on $\mathbb{P}^{n}$ is the class $[s] \in \mathbb{P} \mathbf{E}$ of a global section $s \in \mathbf{E}$. We denote the scheme of zeroes of $[s]$ by $([s])_{0}$. We say $[s]$ has isolated singularities if $\operatorname{dim}([s])_{0}=0$ and we say is non-degenerate, if it has isolated singularities and $([s])_{0}$ is reduced.

It is known that a foliation $[s]$ of degree $r \geq 2$ in $\mathbb{P}^{n}$ with isolated singularities is uniquely determined by $([s])_{0}$, in the sense that $\left(\left[s^{\prime}\right]\right)_{0} \supseteq([s])_{0}$ for some $\left[s^{\prime}\right]$ of degree $r$, implies that $\left[s^{\prime}\right]=[s]$ (that is, $s^{\prime}=\lambda \cdot s$, for some $\lambda \in \mathbf{K}^{*}$ ): For $\mathbf{K}=\mathbb{C}$, the result was first established for $[s]$ non-degenerate, in [10] and the general statement was later obtained in [6]. For an algebraically closed ground field $\mathbf{K}$, it was established for $n=2$ in [5], and the general version was finally established in [1].

Let $[s]$ be a foliation of degree $r>2$ in $\mathbb{P}^{n}$, with isolated singularities. At least if $\mathbf{K}=\mathbb{C}$, there always exist proper subschemes $Z \subset([s])_{0}$ such that $[s]$ is uniquely determined by $Z$ in the sense that $\left(\left[s^{\prime}\right]\right)_{0} \supseteq Z$ for some $\left[s^{\prime}\right]$ of degree $r$, implies that $\left[s^{\prime}\right]=[s]$. This is the content of Proposition 1.1 below. Given $n \geq 2$, and $r \geq 2$, the degree of such subschemes $Z$ is bounded from below by a certain integer $m(n, r-1)$ which we compute in Lemma 1.2 below. The main result of the paper, Theorem 1.3 below, is that the set of foliations $[s]$ (with isolated singularities or not) which are uniquely determined by a $Z \subset([s])_{0}$ having this minimal degree contains a nonempty Zariski open subset of $\mathbb{P E}$.

Our main reference is [3]. Our notation comes from there.

Let $U \subset \mathbb{P}^{n}$ be an open affine that trivializes $\Theta_{\mathbb{P}^{n}}(r-1)$, and let $p \in U$. The restriction of a section $s \in \mathbf{E}$ to $U$ is an affine vector field $\hat{s}=\left(s^{1}, \ldots, s^{n}\right)$. The multiplicity $\mu(s, p)$ of $s$ at $p$, is 
the intersection multiplicity at $p$ of the hypersurfaces $s^{j}=0$, i.e., the vector-codimension in the local ring $\mathcal{O}_{\mathbb{P}^{n}, p}$ of the ideal generated by $\left\{s^{j}\right\}_{j=1}^{n}$ :

$$
\mu(s, p)=\operatorname{dim}_{\mathbf{K}} \mathcal{O}_{\mathbb{P}^{n}, p} /\left(s^{1}, \ldots, s^{n}\right) \cdot \mathcal{O}_{\mathbb{P}^{n}, p} .
$$

It is clear that $\mu(s, p)=\mu(\lambda s, p)$, for every $\lambda \in \mathbf{K}^{*}$, so that $\mu([s], p)=\mu(s, p)$ is well-defined and, moreover, that $p$ is a singularity of $[s]$ if and only if $\mu(s, p) \neq 0$ and that $\mu(s, p)$ is non-zero and finite if and only if $p$ is an isolated singularity of $[s]$. Moreover, $[s]$ is non-degenerate if and only if $\mu([s], p)=1$, for every $p \in([s])_{0}$.

It follows from the Euler sequence

$$
0 \longrightarrow \mathcal{O}_{\mathbb{P}^{n}}(r-1) \longrightarrow \mathcal{O}_{\mathbb{P}^{n}}(r)^{\oplus(n+1)} \stackrel{\Pi_{*}}{\longrightarrow} \Theta_{\mathbb{P}^{n}}(r-1) \longrightarrow 0,
$$

that $\mathbf{e}=(n+1)\left(\begin{array}{c}n+r \\ n\end{array}\right)-\left(\begin{array}{c}n+r-1 \\ n\end{array}\right)$ and that a foliation $[s]$ with isolated singularities has

$$
\operatorname{deg}([s])_{0}=c_{n}\left(\Theta_{\mathbb{P}^{n}}(r-1)\right)=\sum_{j=0}^{n} r^{j}
$$

zeroes, counting multiplicities.

The subsets $\mathcal{U}_{n d} \subset \mathcal{U}_{0}$ of foliations which are non-degenerate resp. have isolated singularities are both non-empty Zariski-open in $\mathbb{P} \mathbf{E}$.

The sheaf of ideals of a closed subscheme $Z \subset \mathbb{P}^{n}$ will be denoted by $I_{Z}$. For a zero-dimensional subscheme $Y \subset \mathbb{P}^{n}$, the space of sections $\mathrm{H}^{0}\left(\mathbb{P}^{n}, \Theta_{\mathbb{P}^{n}} \otimes I_{Y}(r-1)\right)$ that vanish on $Y$ will be denoted by

$$
\mathbf{E}_{Y}=\mathrm{H}^{0}\left(\mathbb{P}^{n}, \Theta_{\mathbb{P}^{n}} \otimes I_{Y}(r-1)\right), \text { with } \mathbf{e}_{Y}=\operatorname{dim}_{\mathbf{K}} \mathbf{E}_{Y}
$$

If $Y$ has degree $y$ and it is reduced, we may consider it as a point in the symmetric product $S^{y} \mathbb{P}^{n}$

Our first result generalizes [7, Corollary 3.3]:

Proposition 1.1. Let $n \geq 2$ and $r \geq 2$ be integers, let $[s]$ be a foliation with isolated singularities of degree $r$ in the complex projective space $\mathbb{P}^{n}$, and let $s_{1} \in H^{0}\left(\mathbb{P}^{n}, \Theta_{\mathbb{P}^{n}}(r-1)\right)$ be a section that vanishes at a subscheme $Z^{\prime} \subset([s])_{0}$ whose degree satisfies $\operatorname{deg} Z^{\prime} \geq \operatorname{deg}([s])_{0}-(n(r-1)-r)$. Then $s_{1}=\lambda s$ for some $\lambda \in \mathbb{C}^{*}$ and hence $\left[s_{1}\right]=[s]$.

Lemma 1.2. Let $n \geq 2$ and $r \geq 2$, be integers, let $\mathbf{e}$ be given by $(1.1)$. Let $\omega=\omega(n, r-1)=\left[\frac{\mathbf{e}-1}{n}\right]$ be the integral part of the number between brackets and let $0 \leq \rho \leq n-1$ be the unique integer such that $\mathbf{e}-1=n \cdot \omega+\rho$.

Let $Y$ be a zero-dimensional closed subscheme of $\mathbb{P}^{n}$ of degree $y$, and assume that

$$
\mathbf{e}_{Y}=h^{0}\left(\mathbb{P}^{n}, \Theta_{\mathbb{P}^{n}} \otimes I_{Y}(r-1)\right)=1 \text {. }
$$

Then $y \geq \omega$, if $\rho=0$ and $y \geq \omega+1$, if $1 \leq \rho \leq n-1$. In consequence, the minimal possible degree $m(n, r-1)$ of a zero-dimensional subscheme $Y \subset([s])_{0}$ such that $\mathbf{e}_{Y}=1$ is

$$
m(n, r-1)= \begin{cases}\omega & \text { if } \rho=0, \text { and } \\ \omega+1 & \text { if } 1 \leq \rho \leq n-1 .\end{cases}
$$

Theorem 1.3. Let $n \geq 2$ and $r \geq 2$, be integers. Let $\omega=\left[\frac{\mathbf{e}-1}{n}\right]$ and $0 \leq \rho \leq n-1$ be as in Lemma 1.2, and $\mathbf{e}_{Y}$, as in (1.4).

(a) If $\rho=0$, then the subset

$$
\mathbf{B}_{\omega}=\left\{[s] \in \mathbb{P} \mathbf{E} \mid \exists Y \in S^{\omega} \mathbb{P}^{n} \text { with } Y \subset([s])_{0} \text { and } \mathbb{P} \mathbf{E}_{Y}=\{[s]\}\right\}
$$

contains a nonempty Zariski-open subset $V_{\omega}$ of $\mathbb{P E}$. 
(b) If $1 \leq \rho \leq n-1$, then the subset

$$
\mathbf{B}_{\omega+1}=\left\{[s] \in \mathbb{P} \mathbf{E} \mid \exists Y^{1} \in S^{\omega+1} \mathbb{P}^{n} \text { with } Y^{1} \subset([s])_{0} \text { and } \mathbb{P} \mathbf{E}_{Y^{1}}=\{[s]\}\right\}
$$

contains a nonempty Zariski-open subset $V_{\omega+1}$ of $\mathbb{P} \mathbf{E}$.

It follows in particular that for $\mathcal{U}=\mathcal{U}_{n d}$ or $\mathcal{U}_{0}$ and $V=V_{\omega}$ or $V_{\omega+1}$ (depending on (a) or (b) above, resp.), the subsets $\mathcal{U} \cap V$ are nonempty Zariski-open subsets of $\mathbb{P} \mathbf{E}$.

\section{THE PROOFS}

Proof of Proposition 1.1. Consider $X=\mathbb{P}^{n}, E=\Theta_{\mathbb{P}^{n}}(r-1), Z=(s)_{0}$ and a fixed divisor $L$ of degree $\ell$ on $\mathbb{P}^{n}$. It is then clear that $\operatorname{det} E=\mathcal{O}_{\mathbb{P}^{n}}(n r+1)$ and that $F$ in the complete linear system $|\operatorname{det} E-L|$ has degree $n r+1-\ell=r+1$ if and only if $\ell=(n-1) r$. Hence, the linear system $\left.\left|K_{\mathbb{P}^{n}}+L\right|=\mathbb{P H}^{0}\left(\mathbb{P}^{n}, \mathcal{O}_{\mathbb{P}^{n}}(\ell-(n+1))\right)=\mathbb{P H}^{0}\left(\mathbb{P}^{n}, \mathcal{O}_{\mathbb{P}^{n}}(n(r-1)-r-1)\right)\right)$ is $(k-1)$-very ample [14, Definition 1.1] if and only if $k=n(r-1)-r$. It follows from [14, Theorem 1.2] that any $F \in|\operatorname{det} E-L|=\mathbb{P H}^{0}\left(\mathbb{P}^{n}, \mathcal{O}_{\mathbb{P}^{n}}(r+1)\right)$ that passes through a subscheme $Z^{\prime} \subset(s)_{0}$ of degree $\operatorname{deg} Z^{\prime} \geq \operatorname{deg}(s)_{0}-(n(r-1)-r)$ necessarily passes through all of $(s)_{0}$. Now, for an $s_{1}$ as in the statement, each of its components satisfy the conditions of the $F$ above, and hence $\left(s_{1}\right)_{0} \supseteq(s)_{0}$. This, together with [6, Theorem 3.5], gives the desired conclusion.

Proof of Lemma 1.2. Let $Y \in S^{y} \mathbb{P}^{n}$ be a zero-dimensional closed subscheme of $\mathbb{P}^{n}$ of degree $y$, with sheaf of ideals $I_{Y}$. We have a short exact sequence of sheaves

$$
0 \longrightarrow I_{Y} \longrightarrow \mathcal{O}_{\mathbb{P}^{n}} \longrightarrow \mathcal{O}_{Y} \longrightarrow 0 \text {. }
$$

It follows that $\mathrm{h}^{2}\left(\mathbb{P}^{n}, I_{Y}(j)\right)=0$, for $n=2$ and $j \geq-2$ from [9, Lemma 2.4] and for $n>2$ and every $j$, from Serre's computations.

Now, consider the short exact sequence obtained by tensoring (1.3) with the sheaf $I_{Y}$ above and its associated long exact cohomology sequence. Using an appropriate twist of (2.1), it follows easily that

$$
\begin{aligned}
& \mathrm{h}^{0}\left(\mathbb{P}^{n}, \Theta_{\mathbb{P}^{n}} \otimes I_{Y}(r-1)\right)-\mathrm{h}^{1}\left(\mathbb{P}^{n}, \Theta_{\mathbb{P}^{n}} \otimes I_{Y}(r-1)\right) \\
& =\mathrm{h}^{0}\left(\mathbb{P}^{n}, \Theta_{\mathbb{P}^{n}}(r-1)\right)-n \cdot y, \text { or } \\
& \mathbf{e}_{Y}-\mathbf{e}_{Y}^{1}=\mathbf{e}-n \cdot y, \text { where } \mathbf{e}_{Y}^{1}=\mathrm{h}^{1}\left(\mathbb{P}^{n}, \Theta_{\mathbb{P}^{n}} \otimes I_{Y}(r-1)\right) .
\end{aligned}
$$

For a closed point $p \in \mathbb{P}^{n}$, the (linear) space $\mathbf{E}_{p}$ has codimension $n$ in $\mathbb{P E}$. Hence, the term $\mathbf{e}_{Y}^{1}=\mathrm{h}^{1}\left(\mathbb{P}^{n}, \Theta_{\mathbb{P}^{n}} \otimes I_{Y}(r-1)\right)$ in $(2.2)$ is equal to the number of dependent conditions imposed by the points of $Y$ in $\mathbf{E}$.

Now assume $\mathbf{e}_{Y}=1$.

It then follows from (2.2) that $n \cdot y-\mathbf{e}_{Y}^{1}=\mathbf{e}-\mathbf{e}_{Y}=\mathbf{e}-1 \leq n \cdot y$, and hence, that $\omega=\left[\frac{\mathbf{e}-1}{n}\right] \leq \frac{\mathbf{e}-1}{n} \leq y$, which is the first assertion. In the same vein, it is easy to see that if $\mathbf{e}_{Y}=1$ and $y=\omega$, then $\mathbf{e}_{Y}^{1}=0$ if and only if $\rho=0$.

On the other hand, if $1 \leq \rho \leq n-1$, then $\mathbf{e}-1=\omega \cdot n+\rho=\mathbf{e}-\mathbf{e}_{Y}=y \cdot n-\mathbf{e}_{Y}^{1}$, hence $(y-\omega) \cdot n=\left(\mathbf{e}_{Y}^{1}-\rho\right)$ : This equation cannot hold for $y=\omega$ and hence $y-\omega \geq 1$, which is the second assertion. We close by recalling that if $y=\omega+1$ and $\mathbf{e}_{Y}=1$, then $\mathbf{e}_{Y}^{1}=n-\rho>0$, as is easy to see.

Proof of Theorem 1.3 (a). This is a straight-forward consequence of [3, Theorem 0.1]. The proof is included to fix our further notation.

For $y \leq \omega=\omega(n, r-1)$, let $N_{y, k}=\left\{Y \in S^{y} \mathbb{P}^{n} \mid \mathbf{e}_{Y}=\mathbf{e}-n \cdot y+k\right\}$. It follows that $N_{y}=N_{y, 0} \subset S^{y} \mathbb{P}^{n}$ is open (because it is the subvariety of $Y \in S^{y} \mathbb{P}^{n}$ where $\mathbf{e}_{Y}$ attains its minimum value) and nonempty (because of [3, Theorem 0.1(a)]). It is hence dense in $S^{y} \mathbb{P}^{n}$ and $\operatorname{dim} N_{y}=n \cdot y$. 
Let

$$
S^{y} \mathbb{P}^{n} \stackrel{\hat{\Pi}_{1}}{\longleftarrow} S^{y} \mathbb{P}^{n} \times \mathbb{P} \mathbf{E} \stackrel{\hat{\Pi}_{2}}{\longrightarrow} \mathbb{P} \mathbf{E} .
$$

be the product variety with canonical projections. Let $A$ be the universal family of foliations of degree $r-1$ from [10], and consider the variety

$$
\begin{aligned}
Z_{y} & =\left\{(Y,[s]) \in S^{y} \mathbb{P}^{n} \times \mathbb{P} \mathbf{E} \mid A(p,[s])=[s](p)=0, \text { for every } p \in Y\right\} \\
& =\left\{(Y,[s]) \in S^{y} \mathbb{P}^{n} \times \mathbb{P} \mathbf{E} \mid p \in([s])_{0}, \text { for every } p \in Y\right\} \\
& =\left\{(Y,[s]) \in S^{y} \mathbb{P}^{n} \times \mathbb{P} \mathbf{E} \mid Y \subset([s])_{0}\right\} \\
& =\left\{(Y,[s]) \in S^{y} \mathbb{P}^{n} \times \mathbb{P} \mathbf{E} \mid[s] \in \mathbb{P} \mathbf{E}_{Y}\right\} \subset S^{y} \mathbb{P}^{n} \times \mathbb{P} \mathbf{E},
\end{aligned}
$$

with restrictions $\Pi_{1}=\left.\hat{\Pi}_{1}\right|_{Z_{y}}: Z_{y} \longrightarrow S^{y} \mathbb{P}^{n}$ and $\Pi_{2}=\left.\hat{\Pi}_{2}\right|_{Z_{y}}: Z_{y} \longrightarrow \mathbb{P E}$. Let

$$
Z_{y, 0}=\Pi_{1}^{-1}\left(N_{y}\right)=\left\{(Y,[s]) \in Z_{y} \mid Y \in N_{y}\right\} .
$$

$Z_{y, 0}$ is open in $Z_{y}$. It is moreover irreducible and has the same dimension

$$
n \cdot y+\mathbf{e}-n \cdot y-1=\mathbf{e}-1
$$

as $\mathbb{P} \mathbf{E}$ does, because all fibers $\Pi_{1}^{-1}(Y)$ are irreducible and have the same dimension

$$
\operatorname{dim} \mathbb{P} \mathbf{E}_{Y}=\mathbf{e}-n \cdot y-1
$$

(which is equal to zero, if $y=\omega$ and $\rho=0$ ). Now consider the restrictions

$$
\Pi_{1}=\left.\Pi_{1}\right|_{Z_{y, 0}}: Z_{y, 0} \longrightarrow N_{y} \subset S^{y} \mathbb{P}^{n}
$$

and $\Pi_{2}=\left.\Pi_{2}\right|_{Z_{y, 0}}: Z_{y, 0} \longrightarrow \mathbb{P E}$ and recall that, set-theoretically,

$$
\boldsymbol{\Pi}_{2}\left(Z_{y, 0}\right)=\left\{[s] \in \mathbb{P} \mathbf{E} \mid \exists Y \in N_{y} \text { such that } Y \subset([s])_{0}\right\} .
$$

$\Pi_{2}$ is a regular map between irreducible varieties of the same dimension which we claim to be dominant (the closure of its image $\overline{\boldsymbol{\Pi}_{2}\left(Z_{y, 0}\right)}$ is $\mathbb{P E}$ or $B=\boldsymbol{\Pi}_{2}\left(Z_{y, 0}\right)$ is contained in no hypersurface). Assuming this for a moment, we may finish the proof applying [11, Proposition 6.4.1] which shows the existence of a subset

$$
V_{y} \subset B
$$

which is open and dense in $\bar{B}=\mathbb{P} \mathbf{E}$ and the desired conclusion follows taking $y=\omega$ in (2.4).

We prove that $\boldsymbol{\Pi}_{2}$ is dominant by contradiction: If it were not, then we may assume that $\bar{B}=C$ is an irreducible hypersurface and there exists a nonempty subset $V^{\prime} \subset B$ open and dense in $C$ such that $\operatorname{dim} \Pi_{2}^{-1}\left(\left[s_{0}\right]\right)=\operatorname{dim} Z_{y, 0}-\operatorname{dim} C=1$, for every $\left[s_{0}\right] \in V^{\prime}$. Since

$$
\Pi_{2}^{-1}\left(\left[s_{0}\right]\right)=\left\{\left(Y,\left[s_{0}\right]\right) \mid Y \in N_{y} \text { and } Y \subset\left(\left[s_{0}\right]\right)_{0}\right\},
$$

the condition $\operatorname{dim} \boldsymbol{\Pi}_{2}{ }^{-1}\left(\left[s_{0}\right]\right)=1$ implies that some $p \in Y$ is a non-isolated singularity of $\left[s_{0}\right]$. Since $V^{\prime}=C \cap W$ for some non-empty open set $W \subset \mathbb{P} \mathbf{E}$, it follows that $\Pi_{2}{ }^{-1}\left(V^{\prime}\right)$ is a nonempty open subset of the irreducible $Z_{y, 0}$, hence it is dense. Consider a $Y \in N_{y}$ from (2.5). Then $\boldsymbol{\Pi}_{2}^{-1}\left(V^{\prime}\right) \cap\left(Y \times \mathbb{P} \mathbf{E}_{Y}\right) \neq \emptyset$ is therefore open and dense. This implies that

$$
\left\{[s] \in \mathbb{P} \mathbf{E}_{Y} \mid \exists p \in Y \text { with } \operatorname{dim}_{p}([s])_{0}=1\right\}
$$

is non-empty and open, therefore is dense in $\mathbb{P} \mathbf{E}_{Y}$.

On the other hand, for $Y \in N_{y}$, the subspace $\mathbf{E}_{Y}$ is the transversal intersection $\bigcap_{p \in Y} \mathbf{E}_{p}$ of the linear subspaces $\mathbf{E}_{p} \subset \mathbf{E}$ and $\mathbf{E}_{p}^{1}=\{s \in \mathbf{E} \mid \mu([s], p)=1\}$ is open and dense in $\mathbf{E}_{p}$ (by [10, Lemma 1.2]), so that

$$
\bigcap_{p \in Y} \mathbf{E}_{p}^{1} \subset \mathbf{E}_{Y} \text { is also open and dense in } \mathbf{E}_{Y}
$$


and

$$
\mathbb{P}\left(\bigcap_{p \in Y} \mathbf{E}_{p}^{1}\right) \subset \mathbb{P} \mathbf{E}_{Y} \text { is open and dense. }
$$

These two open sets of $\mathbb{P E}_{Y}$ should have non-empty intersection, which is clearly absurd. This finishes the proof of Theorem 1.3 (a).

To prepare for the proof of Theorem 1.3 (b), we keep the previously introduced notation. We still consider $y=\omega$ and the unique $\rho$ with $1 \leq \rho \leq n-1$ such that $\mathbf{e}-1=n \cdot \omega+\rho$. It follows that $\mathbf{e}_{Y}=\rho+1$ if $Y \in N_{\omega}$. For each such $Y$, let $\mathbf{s}=\left\{s_{0}, \ldots, s_{\rho}\right\}$ be a $\mathbf{K}$-basis of $\mathbf{E}_{Y}$ : These sections define a vector-bundle map

$$
\phi=\phi_{\mathbf{s}}: \mathbf{T} \longrightarrow \Theta_{\mathbb{P}^{n}}(r-1)
$$

from the trivial vector bundle $\mathbf{T}$ of rank $\rho+1$. In an open affine $U \subset \mathbb{P}^{n}$ that trivializes both $\mathbf{T}$ and $\Theta_{\mathbb{P}^{n}}(r-1), \phi$ is represented by the matrix

$$
A=A_{\mathbf{s}}=\left[\hat{s_{0}}, \ldots, \hat{s_{\rho}}\right] \in M_{n \times(\rho+1)}\left(\mathcal{O}_{\mathbb{P}^{n}}(U)\right)
$$

whose column $\hat{s}_{i}=\left(s_{i}^{1}, \ldots, s_{i}^{n}\right) \in \mathcal{O}_{\mathbb{P}^{n}}(U)^{\oplus n}$ is the restriction of $s_{i}$ to $U$, for $i=0, \ldots, \rho$.

On the other hand, let $\mathbf{M}=M_{n \times(\rho+1)}(\mathbf{K})$ be the affine variety of matrices with $n$ rows and $(\rho+1)$ columns with coefficients in $\mathbf{K}$. It is well-known (see [4]) that the subvariety $\mathbf{M}_{\rho}$ of matrices $A \in \mathbf{M}$ with $\operatorname{rk} A \leq \rho$ is irreducible and has codimension $n-\rho$ in $\mathbf{M}$. This means that the ideal $\mathbf{I}_{\rho}$ of $\mathbf{M}_{\rho}$ is generated by some $n-\rho$ (maximal) minors

$$
A^{J} \subset A \text { of size }|J|=\rho+1,
$$

and $\mathbf{M}_{\rho}$ is (arithmetically) Cohen-Macaulay. The matrix $A_{\mathbf{s}}$ corresponds to a morphism $f: U \longrightarrow \mathbf{M} ; x \mapsto A_{\mathbf{s}}(x)$, and $U_{\rho+1}=f^{-1}\left(\mathbf{M}_{\rho}\right)$ is independent of the trivialization chosen. This allows to define the degeneracy locus $D_{\rho+1}(\mathbf{s})$ of the collection of sections $\mathbf{s}$ by

$$
U_{\rho+1}=D_{\rho+1}(\mathbf{s}) \bigcap U
$$

(see $[2, \mathrm{II}, \S 4])$. It is clear form this construction that

$$
D_{\rho+1}(\mathbf{s})=\left\{p \in \mathbb{P}^{n} \mid\left(s_{0} \wedge \cdots \wedge s_{\rho}\right)(p)=0\right\}, \text { and that } \operatorname{codim} D_{\rho+1}(\mathbf{s}) \leq n-\rho .
$$

Similarly, we have

$$
D_{\rho}(\mathbf{s})=\left\{p \in \mathbb{P}^{n} \mid\left(s_{i_{1}} \wedge \cdots \wedge s_{i_{\rho}}\right)(p)=0, \text { for every }\left\{i_{1}, \ldots, i_{\rho}\right\} \subset\{0, \ldots, \rho\}\right\} .
$$

Our interest in these degeneracy loci comes from the following facts:

Lemma 2.1. Let $n \geq 2$ and $r \geq 2$, and assume that $\mathbf{e}-1 \equiv \rho \bmod n$, with $1 \leq \rho \leq n-1$. Let $Y \in N_{\omega}$ and let $\mathbf{s}=\left\{s_{0}, \ldots, s_{\rho}\right\}$ be a $\mathbf{K}$-basis of $\mathbf{E}_{Y}$. Then $D_{\rho+1}(\mathbf{s})$ is the locus of singular points $p \in \mathbb{P}^{n}$ of sections $s=s_{\lambda} \in \mathbf{E}_{Y}$, and, moreover, $D_{\rho+1}(\mathbf{s}) \backslash D_{\rho}(\mathbf{s})$ is the locus of points $p \in \mathbb{P}^{n}$ such that there exists a unique $[s] \in \mathbb{P} \mathbf{E}_{Y}$ that vanishes both at $Y$ and at $p$.

Proof. Recall that

$$
\begin{aligned}
U_{\rho+1} & =D_{\rho+1}(\mathbf{s}) \bigcap U=\left\{x \in U \mid \operatorname{rk} A_{\mathbf{s}}(x) \leq \rho\right\} \\
& =\left\{x \in U \mid A_{\mathbf{s}}(x) \cdot \vec{\lambda}=\overrightarrow{0}, \text { for some } 0 \neq \vec{\lambda}=\left(\lambda_{0}, \ldots, \lambda_{\rho}\right)^{t} \in \mathbf{K}^{\rho+1}\right\} \\
& =\left\{x \in U \mid \text { there exists } \vec{\lambda} \neq \overrightarrow{0} \in \mathbf{K}^{\rho+1} \text { such that } \hat{s}_{\lambda}(x)=\left(\sum_{i=0}^{\rho} \lambda_{i} \hat{s}_{i}\right)(x)=\overrightarrow{0}\right\} \\
& =\left\{x \in U \mid \hat{s_{\lambda}}(x)=0, \text { for some } s_{\lambda} \in \mathbf{E}_{Y}\right\},
\end{aligned}
$$

which proves the first statement. 
For the second one, recall that $\left(D_{\rho+1}(\mathbf{s}) \backslash D_{\rho}(\mathbf{s})\right) \cap U$ is the set of $x \in U$ such that $\operatorname{rk} A_{\mathbf{s}}(x)=\rho$, so that there exists a unique $\overrightarrow{0} \neq \vec{\lambda}$ (modulo scalar multiplication) such that $s_{\lambda}(x)=0$ for $s_{\lambda}=\sum_{i=0}^{\rho} \lambda_{i} s_{i} \in \mathbf{E}_{Y}$.

Remark 2.2. Under the conditions of Lemma 2.1:

(1) We have $D_{\rho+1}(\mathbf{s}) \backslash D_{\rho}(\mathbf{s}) \neq \emptyset$ : Indeed, if $\left(D_{\rho+1}(\mathbf{s}) \backslash D_{\rho}(\mathbf{s})\right) \cap U=\emptyset$ for every such $U$, then the sections in $\mathbf{s}$ are linearly dependent in all $\mathbb{P}^{n}$ and hence, they form no basis of $\mathbf{E}_{Y}$, which is absurd.

(2) It is easy to see that, for $\tau=\rho$ and $\rho+1$, we have $D_{\tau}(\mathbf{s})=D_{\tau}\left(\mathbf{s}^{\prime}\right)$, for any other $\mathbf{K}$-basis $\mathbf{s}^{\prime}$ of $\mathbf{E}_{Y}$. This allows us to define $D_{\tau}(Y)$ to be $D_{\tau}(\mathbf{s})$, for some $\mathbf{K}$-basis $\mathbf{s}$ of $\mathbf{E}_{Y}$, and

$$
C_{Y}=D_{\rho+1}(Y) \backslash D_{\rho}(Y) \text {. }
$$

Hence, $C_{Y}$ is nonempty for every $Y \in N_{\omega}$ and it follows from Lemma 2.1 that, for every $Y^{1}=Y \times\{p\} \in N_{\omega} \times C_{Y} \subset S^{\omega+1} \mathbb{P}^{n}$, there exists a unique $[s] \in \mathbb{P E}_{Y^{1}}$. It follows moreover that

$$
\operatorname{dim} C_{Y}=\operatorname{dim} D_{\rho+1}(Y), \text { for every } Y \in N_{\omega} .
$$

We have the following refinement of [2, II§4 Proposition 4.1]:

Proposition 2.3. Let $n \geq 2$ and $r \geq 2$ be integers such that

$$
\mathbf{e}-1 \equiv \rho \bmod n, \quad \text { with } 1 \leq \rho \leq n-1
$$

and let $Y \in N_{\omega}$. Then, $D_{\rho+1}(Y)$ has the expected codimension $n-\rho$ and hence it is a complete intersection. In consequence, it is not only (arithmetically) Cohen-Macaulay, but also equidimensional of dimension $\rho$. In particular, $C_{Y}$ is equidimensional of dimension $\rho$.

Proof. Let $\mathbf{s}=\left\{s_{0}, \ldots, s_{\rho}\right\}$ be a $\mathbf{K}$-basis of $\mathbf{E}_{Y}$. On the one hand, it follows from (2.6) that we may assume that $\mu\left(\left[s_{i}\right], p\right)=1$, for every $p \in Y$ and every $i=0, \ldots, \rho$. On the other hand, consider the matrix $A_{\mathbf{s}}(x)$ from (2.7), with $x$ in some such $U \subset \mathbb{P}^{n}$. For $J=\left\{j_{1}<\cdots<j_{\rho+1}\right\}$, let

$$
A_{\mathbf{s}}(x)^{J}=\left(\begin{array}{ccc}
s_{0}^{j_{1}}(x) & \cdots & s_{\rho}^{j_{1}}(x) \\
\vdots & \ddots & \vdots \\
s_{0}^{j_{\rho+1}}(x) & \cdots & s_{\rho}^{j_{\rho+1}}(x)
\end{array}\right)
$$

be a (maximal) minor of $A_{\mathbf{s}}(x)$ from (2.8). We have already seen in (2.9) that

$$
\operatorname{codim} D_{\rho+1}(Y) \leq n-\rho .
$$

If $\rho=n-1$, then it is clear that $D_{n}(Y)$ is a hypersurface, so we can assume that $\rho<n-1$ : Assume that $\operatorname{codim} D_{\rho+1}(Y)$ is strictly smaller than $n-\rho$, say, equal to $n-\rho-1$, then one of these (maximal) minors $A_{\mathbf{s}}(x)^{J}$ has determinant identically equal to zero and hence, at least one of its rows is linearly dependent to the others. This implies that, for every $s_{i} \in \mathbf{s}$, no $p \in Y \cap U$ is an isolated singularity of $s_{i}$, because $\left(s_{i, p}^{1}, \ldots, s_{i, p}^{n}\right) \subset \mathcal{O}_{\mathbb{P}^{n}, p}$ is not a regular sequence. This contradiction shows that $\operatorname{codim} D_{\rho+1}(Y)=n-\rho$. The Cohen-Macaulay and equidimensionality properties of $D_{\rho+1}(Y)$ follow from [8] (Proposition 18.13 and Corollary 18.14, respectively). The last statement is clear from Remark 2.2.

Proof of Theorem 1.3 (b). In analogy with (2.3), let

$$
Z_{\omega+1}=\left\{\left(Y^{1},[s]\right) \in S^{\omega+1} \mathbb{P}^{n} \times \mathbb{P} \mathbf{E} \mid Y^{1} \subset([s])_{0}\right\} \subset S^{\omega+1} \mathbb{P}^{n} \times \mathbb{P} \mathbf{E},
$$


with restrictions $\Pi_{1}: Z_{\omega+1} \longrightarrow S^{\omega+1} \mathbb{P}^{n}$ and $\Pi_{2}: Z_{\omega+1} \longrightarrow \mathbb{P E}$. Consider

$$
\begin{aligned}
\widetilde{N}_{\omega+1} & =\left\{Y^{1}=Y \times\{p\} \mid Y \in N_{\omega} \text { and } p \in D_{\rho+1}(Y)\right\} \subset S^{\omega+1} \mathbb{P}^{n}, \\
\widetilde{N}_{\omega+1}^{\prime} & =\left\{Y^{1}=Y \times\{p\} \mid Y \in N_{\omega} \text { and } p \in D_{\rho}(Y)\right\} \subset \widetilde{N}_{\omega+1}, \text { and } \\
M_{\omega+1} & =\left\{Y^{1}=Y \times\{p\} \mid Y \in N_{\omega} \text { and } p \in C_{Y}\right\} \subset \widetilde{N}_{\omega+1} .
\end{aligned}
$$

Let

$$
\widetilde{Z}_{\omega+1}=\Pi_{1}^{-1}\left(\widetilde{N}_{\omega+1}\right) \subset Z_{\omega+1}
$$

and let $\widetilde{\Pi}_{1}: \widetilde{Z}_{\omega+1} \longrightarrow \widetilde{N}_{\omega+1}$ and $\widetilde{\Pi}_{2}: \widetilde{Z}_{\omega+1} \longrightarrow \mathbb{P E}$ be the restrictions to $\widetilde{Z}_{\omega+1}$ of the projections $\Pi_{1}$ and $\Pi_{2}$ above, respectively.

$\widetilde{N}_{\omega+1}$ is a nonempty quasiprojective subvariety, possibly reducible (for $D_{\rho+1}(Y)$ may have components), singular (for $D_{\rho+1}(Y)$ is singular along $D_{\rho}(Y)$ ), but equidimensional of dimension equal to $n \cdot \omega+\rho=\mathbf{e}-1$.

We claim that, set-theoretically:

$$
\widetilde{\Pi}_{2}\left(\widetilde{Z}_{\omega+1}\right)=\Pi_{2}\left(Z_{\omega, 0}\right) \supset V_{\omega} \neq \emptyset,
$$

and in consequence, $\widetilde{\Pi}_{2}$ is dominant (because $\overline{V_{\omega}}=\mathbb{P} \mathbf{E}$ ).

It only remains to prove the equality in the claim and this goes as follows: $[s] \in \mathbf{\Pi}_{2}\left(Z_{\omega, 0}\right)$ if and only if $[s]=\Pi_{2}(Y,[s])$, for some $Y \in N_{\omega}$ and $Y \subset([s])_{0} \subset D_{\rho+1}(Y)$ by Lemma 2.1. In particular, any $q \in([s])_{0} \backslash Y$ lies in $D_{\rho+1}(Y)$ so that $[s]=\widetilde{\Pi}_{2}(Y \times\{q\}$, $[s])$, with $Y^{1}=Y \times\{q\} \in \widetilde{N}_{\omega+1}$. The reciprocal inclusion is trivial.

We claim moreover that there exist

$$
[s] \in V_{\omega} \text { such that }[s]=\widetilde{\Pi}_{2}(Y \times\{p\},[s]) \text {, with } p \in C_{Y}\left(Y \in N_{\omega}\right) .
$$

Otherwise, for every $[s] \in V_{\omega},[s]=\widetilde{\Pi}_{2}(Y \times\{q\},[s])$, for some $q \in D_{\rho}(Y)$ and the restriction $\mathrm{r}_{2}$ of $\widetilde{\Pi}_{2}$ to $\widetilde{\Pi}_{1}^{-1}\left(\widetilde{N}_{\omega+1}^{\prime}\right)$ :

$$
\widetilde{Z}_{\omega+1} \supset \widetilde{\Pi}_{1}^{-1}\left(\widetilde{N}_{\omega+1}^{\prime}\right) \stackrel{\mathrm{r} \widetilde{\Pi}_{2}}{\longrightarrow} \mathbb{P} \mathbf{E}
$$

is dominant. This is absurd, for $\operatorname{dim} \widetilde{\Pi}_{1}^{-1}\left(\widetilde{N}_{\omega+1}^{\prime}\right)<\mathbf{e}-1$, by Remark 2.2, and (2.11) follows.

Finally by the moment, we claim that

$$
\mu\left(\left[s_{0}\right], q\right)=1 \text {, for every }\left[s_{0}\right] \text { and } q \in Y \times\{p\} \in M_{\omega+1} \text { satisfying (2.11) : }
$$

It follows from Lemma 2.1 that $\left[s_{0}\right]$ is the unique foliation that vanishes at every

$$
q \in Y^{1}=Y \times\{p\}
$$

so that $\left\{\left[s_{0}\right]\right\}=\mathbb{P} \mathbf{E}_{Y^{1}} \subset \mathbb{P} \mathbf{E}$ is zero-dimensional. By [10, Lemma 1.2],

$$
\left\{[s] \in \mathbb{P} \mathbf{E}_{Y^{1}} \mid \mu\left(\left[s_{0}\right], q\right)>1, \text { for some } q \in Y^{1}\right\}
$$

is a proper closed subset of $\mathbb{P} \mathbf{E}_{Y^{1}}$, hence is empty, and the conclusion follows.

Now, let

$$
\widetilde{Z}_{\omega+1}^{1}=\widetilde{\Pi}_{1}^{-1}\left(M_{\omega+1}\right) \subset \widetilde{Z}_{\omega+1}
$$

(which is non-empty by $(2.11)$ ) and let $\widetilde{\boldsymbol{\Pi}}_{1}: \widetilde{Z}_{\omega+1}^{1} \longrightarrow M_{\omega+1}$ and $\widetilde{\boldsymbol{\Pi}}_{\mathbf{2}}: \widetilde{Z}_{\omega+1}^{1} \longrightarrow \mathbb{P} \mathbf{E}$ be the restrictions to $\widetilde{Z}_{\omega+1}^{1}$ of the projections $\widetilde{\Pi}_{1}$ and $\widetilde{\Pi}_{2}$.

The quasiprojective variety $M_{\omega+1}$ is non-empty, possibly reducible but equidimensional of dimension equal to $\mathbf{e}-1$, by Proposition 2.3. Moreover, $\widetilde{\mathbf{\Pi}}_{\mathbf{1}}$ is surjective and for every $Y^{1} \in M_{\omega+1}$, the fibre $\widetilde{\boldsymbol{\Pi}}_{\mathbf{1}}^{-1}\left(Y^{1}\right)$ has dimension zero (it is a closed point $\left(Y^{1},[s]\right)$ because $[s]$ is unique, by 
Lemma 2.1). This, together with [13, Theorem 1.26], shows that for every irreducible component $M_{\omega+1}^{c}$ of $M_{\omega+1}$,

$$
\widetilde{Z}_{\omega+1}^{1, c}=\widetilde{\Pi}_{\mathbf{1}}^{-1}\left(M_{\omega+1}^{c}\right) \subset \widetilde{Z}_{\omega+1}^{1}
$$

is irreducible and has dimension $\mathbf{e}-1$. It is hence a component of $\widetilde{Z}_{\omega+1}^{1}$.

Now consider a component $\widetilde{Z}_{\omega+1}^{1, c}$ containing a $(Y \times\{p\},[s])$ satisfying $(2.11)$ and let

$$
\widetilde{\Pi}_{2}^{\mathbf{c}}: \widetilde{Z}_{\omega+1}^{1, c} \longrightarrow \mathbb{P E}
$$

be the restriction to $\widetilde{Z}_{\omega+1}^{1}$ of the map $\widetilde{\boldsymbol{\Pi}}_{\mathbf{2}}$. As with part (a), the proof of Theorem 1.3 (b) follows if we prove that $\widetilde{\mathbf{\Pi}}_{\mathbf{2}}^{\mathbf{c}}$ is dominant for, in this situation [11, Proposition 6.4.1] gives the existence of the Zariski-open set we are seeking for: $V_{\omega+1} \subset \widetilde{\Pi}_{\mathbf{2}}^{\mathbf{c}}\left(\widetilde{Z}_{\omega+1}^{1, c}\right)$, open and dense in $\mathbb{P} \mathbf{E}$, the closure of $\widetilde{\boldsymbol{\Pi}}_{\mathbf{2}}^{\mathbf{c}}\left(\widetilde{Z}_{\omega+1}^{1, c}\right)$.

We prove that $\widetilde{\boldsymbol{\Pi}}_{\mathbf{2}}^{\mathbf{c}}$ is dominant modifying the previous proof that $\boldsymbol{\Pi}_{2}: Z_{y, 0} \longrightarrow \mathbb{P} \mathbf{E}$ is dominant, this time letting $B=\widetilde{\boldsymbol{\Pi}}_{\mathbf{2}}^{\mathbf{c}}\left(\widetilde{Z}_{\omega+1}^{1, c}\right):$ If it were not, then we may assume that $\bar{B}=C$ is an irreducible hypersurface and there exists a nonempty subset $V^{\prime} \subset B$ open and dense in $C$ such that $\operatorname{dim}\left(\widetilde{\boldsymbol{\Pi}}_{\mathbf{2}}^{\mathbf{c}}\right)^{-1}\left(\left[s_{0}\right]\right)=\operatorname{dim} \widetilde{Z}_{\omega+1}^{1, c}-\operatorname{dim} C=1$, for every $\left[s_{0}\right] \in V^{\prime}$. Since

$$
\left(\widetilde{\boldsymbol{\Pi}}_{\mathbf{2}}^{\mathbf{c}}\right)^{-1}\left(\left[s_{0}\right]\right)=\left\{\left(Y^{1},\left[s_{0}\right]\right) \mid Y^{1} \in M_{\omega+1} \text { and } Y^{1} \subset\left(\left[s_{0}\right]\right)_{0}\right\},
$$

the condition $\operatorname{dim}\left(\widetilde{\mathbf{\Pi}}_{\mathbf{2}}^{\mathbf{c}}\right)^{-1}\left(\left[s_{0}\right]\right)=1$ implies that some $p \in Y^{1}$ is a non-isolated singularity of $\left[s_{0}\right]$, for every $\left[s_{0}\right] \in V^{\prime}$.

But on the other hand, these $\left[s_{0}\right]$ certainly do satisfy $(2.11)$ for some $Y^{1}=Y \times\{p\} \in M_{\omega+1}$ and hence, they also must satisfy (2.12). This contradiction shows that (2.13) is dominant and the proof of Theorem 1.3 (b) has been completed.

\section{Closing Remarks}

For $n=2$ and $r \geq 2$, let $M_{r}=r(r+5) / 2$. It is easy to see that

$$
m(2, r-1)=M_{r}-(t-1), \text { either if } r=2 t \text { or } 2 t+1 .
$$

Recall form [7, Theorem 3.5] that for every non-degenerate foliation $[s]$ of degree $r$ in $\mathbb{P}^{2}$, there exists a subscheme $Z \subset([s])_{0}$ of degree $M_{r}$ which determines $[s]$ uniquely (although $\mathbf{K}=\mathbb{C}$ in [7], the attentive reader will notice that the results therein hold for an algebraically closed ground field $\mathbf{K}$ ).

For small values of $r$, we have the following values:

\begin{tabular}{c|ccc}
$r$ & $m(2, r-1)$ & $M_{r}$ & $c_{2}\left(\Theta_{\mathbb{P}^{2}}(r-1)\right)$ \\
\hline 2 & 7 & 7 & 7 \\
3 & 12 & 12 & 13 \\
4 & 17 & 18 & 21 \\
5 & 24 & 25 & 31 \\
6 & 31 & 33 & 43 \\
7 & 40 & 42 & 57
\end{tabular}

We conclude that for a non-degenerate foliation $[s]$ of degree 2 in $\mathbb{P}^{2}$ no proper subscheme of $([s])_{0}$ may uniquely determine $[s]$ and that every non-degenerate foliation $[s]$ of degree 3 in $\mathbb{P}^{2}$ has a minimal subscheme which uniquely determines it.

At this point, we can moreover prove (see [12]) that for any such $Z \subset([s])_{0}$, there exists a subscheme of degree $M_{r}-1$ of $Z$ which still determines $[s]$ uniquely. The conclusion is that every 
non-degenerate foliation $[s]$ of degrees 4 and 5 in $\mathbb{P}^{2}$ has a minimal subscheme which uniquely determines it.

The question wether every non-degenerate foliation $[s]$ of degree $r \geq 6$ in $\mathbb{P}^{2}$ has a minimal subscheme which uniquely determines it remains open.

\section{REFERENCES}

1. C. Araujo, M. Corrêa. On degeneracy schemes of maps of vector bundles and applications to holomorphic foliations, Math. Z. 276 (2014), no. 1-2, 505-515.

2. E. Arbarello, M. Cornalba, P. A. Griffiths, J. Harris. Geometry of algebraic curves. Vol. I Grundlehren der Mathematischen Wissenschaften [Fundamental Principles of Mathematical Sciences], 267. Springer-Verlag, New York, (1985). DOI: 10.1007/978-1-4757-5323-3

3. E. Ballico. On meromorphic vector fields on projective spaces, Amer. J. Math. 115 (1993), no. 5, 1135-1138.

4. W. Bruns, U. Vetter. Determinantal rings. Lecture Notes in Mathematics 1327. Springer-Verlag, Berlin (1988).

5. A. Campillo, J. Olivares, Polarity with respect to a foliation and Cayley-Bacharach Theorems, J. reine angew. Math. 534 (2001), 95-118. DOI: 10.1515/crll.2001.036

6. A. Campillo, J. Olivares, On sections with isolated singularities of twisted bundles and applications to foliations by curves, Math. Res. Lett. 10, (2003) 651-658. DOI: 10.4310/MRL.2003.v10.n5.a8

7. A. Campillo, J. Olivares, Special subschemes of the scheme of singularities of a plane foliation, C. R. Math. Acad. Sci. Paris 344 (2007), no. 9, 581-585.

8. D. Eisenbud, Commutative algebra. With a view toward algebraic geometry. Graduate Texts in Mathematics, 150. Springer-Verlag, New York, (1995).

9. E. Esteves, S. Kleiman, Bounds on leaves of foliations in the plane, Real and complex singularities, Contemp. Math., 354 (2004), 57-67. DOI: 10.1090/conm/354/06474

10. X. Gómez-Mont, G. Kempf, Stability of meromorphic vector fields in projective spaces. Comm. Math. Helv. 64 (1989), 462-473.

11. G. Kempf, Algebraic varieties. London Mathematical Society Lecture Note Series, 172. Cambridge University Press, Cambridge (1993).

12. J. Olivares, Foliations in the plane uniquely determined by minimal subschemes of its singularities, Submitted.

13. I. Shafarevich, Basic algebraic geometry 1. Varieties in projective space. Third edition. Translated from the 2007 third Russian edition. Springer, Heidelberg, (2013).

14. S.-L. Tan. Cayley-Bacharach property and k-very ampleness. First International Congress of Chinese Mathematicians (Beijing, 1998), 241-250, AMS/IP Stud. Adv. Math. 20, Amer. Math. Soc., Providence, RI (2001).

Antonio Campillo, imuVa (Instituto de Investigación en Matemáticas), Universidad de Valladolid, Paseo de Belén, 7, 47011 Valladolid, Spain

Email address: campillo@agt.uva.es

Jorge Olivares, Centro de Investigación en Matemáticas, A.C. A.P. 402, Guanajuato 36000, Mexico. Email address: olivares@cimat.mx 